\title{
Improvement of Power System Stability using BBO Algorithm
}

\author{
Tawfik Guesmi \\ College of Engineering, University of Hail, \\ Hail, Saudi Arabia
}

\begin{abstract}
In this study, a BBO based multimachine power system stabilizers (PSSs) design is proposed for the enhancement of the system damping of low-frequency oscillations. All PSSs parameters are tuned in order to shift all electromechnical modes into the left side of a pre-specified vertical line in the s-plane. The PSS design is formulated as an optimization problem with two eigenvalue based objective functions. A mutation operator is embedded into the BBO algorithm to improve the diversity of solutions. The proposed technique is tested on the 10-machine 39bus New England power system under several operating conditions and configurations. Eigenvalue analysis and nonlinear simulations show that this technique is robust and gives better results as compared with the $\mathrm{ABC}$ algorithm.
\end{abstract}

Keywords- Power System Stabilizers, Low-frequency oscillations, Electromechanical modes, BBO Algorithm

\section{INTRODUCTION}

In recent years, enhancement of power system stability is becoming one of the most important problems encountered by electrical engineers due to the large scale and high nonlinearity of the today's systems. Generally, low frequency oscillations $(0.2-2.5 \mathrm{~Hz})$ due to the sudden variation in the load and/or in the system configuration are the main causes of power system instabilities [1-3]. In fact, if no adequate damping is applied, these oscillations can lead to the system separation and loss of synchronism between generators. Conventional power system stabilizers (CPSS) have been considered for many years as effective controllers in the improvement of the system damping of low-frequency electromechanical oscillations. These stabilizers extend the stability limits by controlling the generator excitation systems [1-5]. The lead-lag compensator based stabilizers are widely used by power system utilities $[1,3]$. However, robustness of these controllers is highly dependent to the compensator parameters. Thus, several techniques have been proposed in the last decades to tune PSS parameters [1-17].

Several conventional techniques such as, sensitivity analysis [9-10], root locus [11], nonlinear optimization and mathematical programming [12] have been proposed. However, these techniques have been criticized for their consuming time. In addition, conventional techniques are iterative and require an initialization step that highly dependent to the operating conditions.

To overcome these drawbacks, several metaheuristic methods have been used for solving the problem of PSS design in recent years. A genetic algorithm (GA) based optimal design of multimachine PSS has been suggested in [13] to maximize the damping performance of low frequency oscillations. Other evolutionary computation technique called particle swarm optimization (PSO) has been applied also in several works for optimum determination of PSS parameters [14-15]. A tabu search (TS) technique for optimum PSS design is presented in [16]. Parameters of all PSSs have been tuned in order to shift all electromechanical modes to the left side of a pre-specified vertical line in the s-plane. Unfortunately, one of the drawbacks of TS algorithm is the tendency to fall into local optimization [18]. In [17], artificial bee colony (ABC) based method has been proposed for PSS tuning where the overshoot and settling time at several loading conditions have been minimized. However, like some other evolutionary algorithms, the $\mathrm{ABC}$ method has also been criticized to its poor convergence rate and premature convergence due to the unbalanced explorationexploitation processes. In recent years, a new naturally inspired algorithm called biogeography-based optimization (BBO) firstly proposed by Simon [19] has demonstrated a high potential in solving several engineering problems. Within this context, this study presents a BBO based method for optimal design of multimachine PSSs. All PSS parameters are tuned in order to shift all undamped and lightly damped electromechanical modes for several operating conditions into a pre-specified zone in the s-plane. In order to enhance the exploration ability and improve the diversity of the population of $\mathrm{BBO}$, a mutation operator is embedded into $\mathrm{BBO}$.

To test the robustness of the extended BBO algorithm for PSS design, this technique is compared with ABC algorithm. Nonlinear time domain simulations carried out on the 10machine 39-bus New England power system show that the proposed method provides a robust performance for a wide range of loading conditions and under several large disturbances.

\section{PROBLEM FORMULATION}

In this study, the third order machine model is used for the PSS design. The widely used CPSS with lead-lag compensator shown in Fig. 1. Thus, the $i$-th synchronous machine equipped with PSS can be described by the following differential equations. All symbols in equations (1)-(4) are defined in [3].

$$
\begin{gathered}
p \delta_{i}=\omega_{b}\left(\omega_{i}-1\right) \\
p \omega_{i}=\left(T_{m i}-T_{e i}-D_{i}\left(\omega_{i}-1\right)\right) / M_{i} \\
p E_{q i}^{\prime}=\left(E_{f d i}-\left(x_{d i}-x_{d i}^{\prime}\right) i_{d i}-E_{q i}^{\prime}\right) / T_{d o i}^{\prime} \\
p E_{f d_{i}}=\left(K_{A_{i}}\left(V_{r e f_{i}}-V_{i}+U_{i}\right)-E_{f d_{i}}\right) / T_{A_{i}}
\end{gathered}
$$




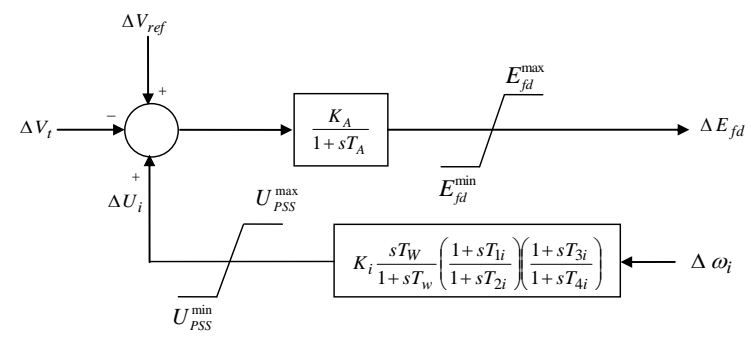

Fig. 1. IEEE Type-ST1 excitation system with PSS

In the design of PSS controllers, the linearized incremental model around the steady state point is widely employed [3]. Unstable electromechanical modes and/or lightly damped modes are among the main causes of large oscillations and instability of electric power systems. Thus, it is well advised to shift these modes into the left side in the s-plane. Within this context, two eigenvalue based objective functions can be considered as follows.

$$
\begin{aligned}
& J_{1}=\sum_{j=1}^{N C} \sum_{\sigma_{i j} \geq \sigma_{0}}\left(\sigma_{0}-\sigma_{i j}\right)^{2} \\
& J_{2}=\sum_{j=1}^{N C} \sum_{\xi_{i j} \leq \xi_{0}}\left(\zeta_{0}-\xi_{i j}\right)^{2}
\end{aligned}
$$

Where, $\sigma_{i j}$ and $\xi_{i j}$ are the real part and the damping ratio of the $i$-th eigenvalue of the $j$-th operating condition.

Within this scope, the optimum PSS design presented in this study aims to minimize $J_{1}$ and $J_{2}$ using the BBO algorithm. This bi-objective optimization is converted into single objective problem as given in the following equation. The problem constraints are the PSSs parameter limits. Since $J_{2}$ is ten times less than $J_{1}$, the weighting coefficient $\alpha$ is a random value between 1 and 10 .

$$
\left\{\begin{array}{l}
\text { Minimize }\left(J=J_{1}+\alpha J_{2}\right) \\
K_{i}^{\min } \leq K_{i} \leq K_{i}^{\max } \\
T_{1 i}^{\min } \leq T_{1 i} \leq T_{1 i}^{\max } \\
T_{2 i}^{\min } \leq T_{2 i} \leq T_{2 i}^{\max } \\
T_{3 i}^{\min } \leq T_{3 i} \leq T_{3 i}^{\max } \\
T_{4 i}^{\min } \leq T_{4 i} \leq T_{4 i}^{\max }
\end{array}\right.
$$

\section{PROPOSED BBO ALGORITHM WITH MUTATION}

Biogeography based optimization (BBO) firstly introduced by Simon [19], is a new algorithm inspired from the principle of movement of species that depends mainly on topographic features of the considered space called habitat and time. The migration of species towards a habitat called immigration, and migration from a habitat called emigration are explained in Fig. 2 . It can be seen that the immigration rate $\lambda$ is equal to its maximum value $I$ when no species in the habitat and it decreases when the habitat will be more crowded. The immigration rate will be zero when the habitat capacity $S_{\max }$ is reached. Moreover, the emigration rate $\mu$ is zero for empty habitat. On the other hand, spices emigrate when the habitat will be crowded to find other suitable residences. The spices emigration rate reaches its maximum value $E$ when the number of spices in the habitat $S$ is equal to $S_{\max }$.

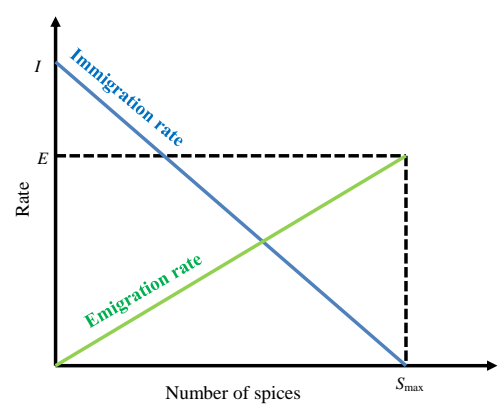

Fig. 2. Migration rates vs. number of spices

For each habitat, a variable called habitat suitability index (HSI) is assigned. Habitats with high HSI that are well suited for residence of spices are more crowded. Thus, more than HSI of the habitat increases more than the immigration rate decreases and emigration rate increases, and vice versa.

Similarly to GA, BBO is a population based technique. Similarities and dissimilarities between GA's and BBO's characteristics have been investigated in [20].

Individuals that are represented by chromosomes in GA are represented by habitats in $\mathrm{BBO}$. The fitness of each candidate habitat is its HSI. Good solutions correspond to habitats having high HSIs. Like GA, BBO has two main operators that are mutation and migration operators. Migration includes emigration and immigration.

\section{A. Migration operators}

Immigration and emigration operators are used to provide enhanced solution for the optimization problem.

Let consider $N=S_{\max }$. According to Fig. 2, the immigration and emigration rates for $\mathrm{k}$ spices in the habitat will be expressed respectively by the following equations.

$$
\begin{gathered}
\lambda_{k}=I\left(1-\frac{k}{N}\right) \\
\mu_{k}=\frac{E k}{N}
\end{gathered}
$$

In BBO algorithm, it is assumed that each solution $X=\left(x_{1}, x_{2}, \ldots, x_{n}\right)$ is considered as habitat. Where $n$ is number of decision variables. These variables characterize habitability feature called suitability index variables (SIVs). For each decision variable is affected an SIV. All solutions will be modified with a pre-specified probability $P_{\text {mod }}$. All SIVs of the solution to be modified will migrate according to the immigration rate of the corresponding habitat. A normalized immigration rate as given in equation (10) can be used. Once SIV is selected to migrate, the emigration rate is used to determine which of the other solutions should migrate its SIV to the solution to be modified. At an iteration $t$, the flowchart of the migration operator is described in Fig. 3.

$$
\lambda_{k} \leftarrow \lambda^{l}+\frac{\left(\lambda^{u}-\lambda^{l}\right)\left(\lambda_{k}-\lambda^{\min }\right)}{\left(\lambda^{\max }-\lambda^{\min }\right)}
$$


Where, $\lambda^{\min }$ and $\lambda^{\max }$ are minimum and maximum bounds of the immigration rate, respectively. $\lambda^{l}$ and $\lambda^{u}$ are lower and upper limits of the normalized immigration rate, respectively.

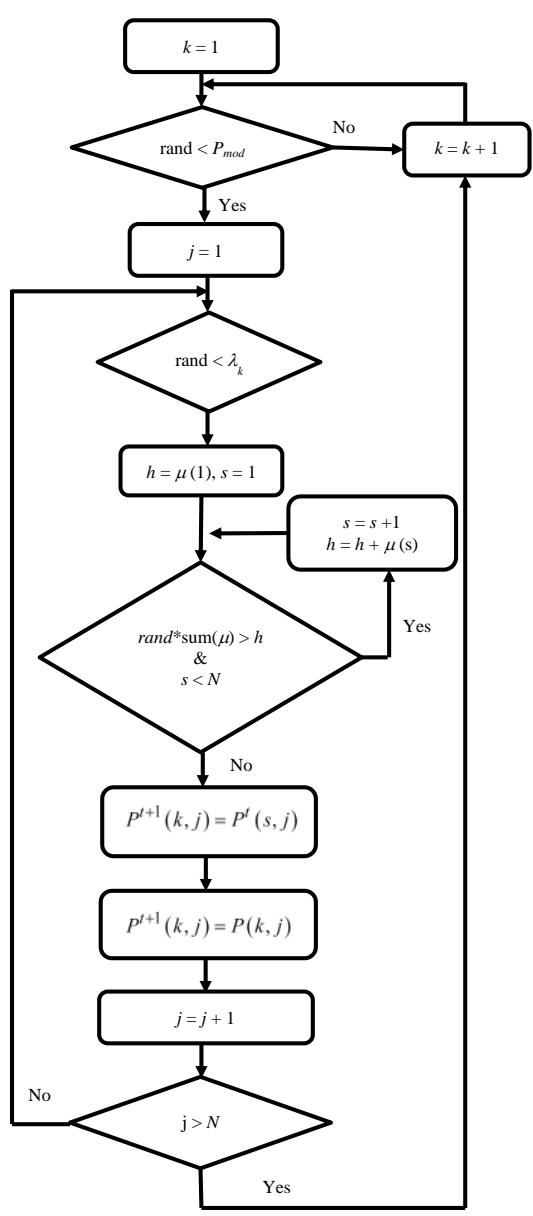

Fig. 3. Flowchart of the migration operator

\section{B. Mutation operator}

HSI of each habitat may undergo drastic change due to climate change, disease, Natural disasters, sudden immigration of large number of species from a neighboring habitat, etc. In $\mathrm{BBO}$ algorithm, this random change is modeled by mutation operator. SVIs of amount of habitats from the population obtained after applying migration operators will be modified using mutation operator according to the mutation rate [21]. As in GA, mutation is applied to ensure diversity of the population in the next iteration. In the most BBO-based optimization techniques, the mutation rate $m_{s}$ for each habitat $H$ depends on the probability $P_{s}$ of that habitat to contain $S$ species. As given in [19], $P_{s}$ is updated for each time step $\Delta t$ as follows.

$$
\begin{aligned}
P_{s}(t+\Delta t)= & P_{s}(t)\left(1-\lambda_{s} \Delta t-\mu_{s} \Delta t\right) \\
& +P_{s-1} \lambda_{s-1} \Delta t+P_{s+1} \mu_{s+1} \Delta t
\end{aligned}
$$

For $\Delta t \rightarrow 0$, equation (11) can provide the following expression.

$$
\dot{P}_{S}=\left\{\begin{array}{lc}
-\left(\lambda_{s}+\mu_{s}\right) P_{s}+\mu_{s+1} P_{s+1} ; & S=0 \\
-\left(\lambda_{s}+\mu_{s}\right) P_{s}+\lambda_{s-1} P_{s-1}+\mu_{s+1} P_{s+1} ; & 1 \leq S \leq S_{\max }-1 \\
-\left(\lambda_{s}+\mu_{s}\right) P_{s}+\lambda_{s-1} P_{s-1} ; & S=S_{\max }
\end{array}\right.
$$

The mutation rate can be described as follows.

$$
m_{s}=m_{\max }\left(1-\frac{P_{s}}{P_{\max }}\right)
$$

Where $m_{\max } \in[0,1]$ is a pre-specified parameter. $P_{\text {max }}=\max \left\{P_{1}, P_{2}, \ldots, P_{N}\right\}$.

In this study, the non-uniform mutation operator has been employed. So, at the $t$-th iteration, each SIV will be transformed to other SIV' with a probability as follows.

$$
\begin{gathered}
S I V^{\prime}=\left\{\begin{array}{l}
S I V+\Delta(t, b-S I V), \text { if } \tau=0 \\
S I V-\Delta(t, S I V-a), \text { if } \tau=1
\end{array}\right. \\
\Delta(t, y)=y\left(1-r^{\left.\left(1-\frac{t}{t_{\max }}\right)^{\beta}\right)}\right.
\end{gathered}
$$

Where $\tau$ is a binary number, $r$ is a random number and $t_{\max }$ is the maximum number of iteration. $a$ and $b$ are lower and upper bounds of the corresponding SIV. $\beta$ represents the dependency degree on the iteration number.

The flowchart of the proposed BBO algorithm with mutation operator is given in Fig. 4.

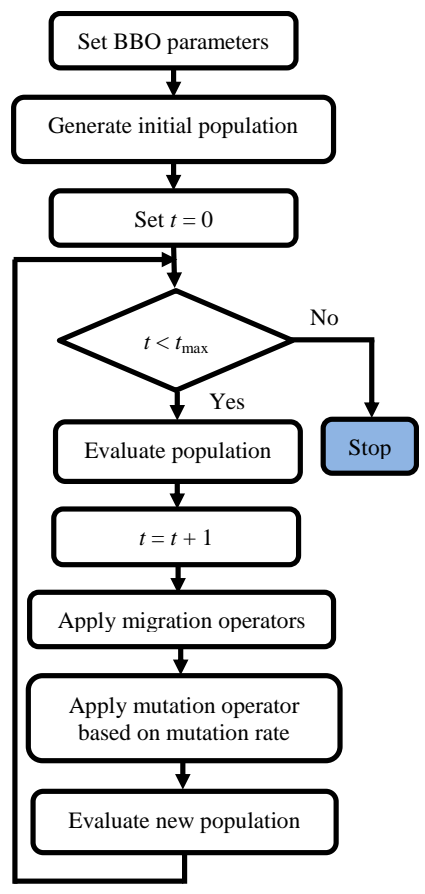

Fig. 4. Flowchart of the proposed optimization algorithm 


\section{SIMULATION RESULTS}

To evaluate the performance of the proposed $\mathrm{BBO}$ based PSS controllers (BBO-PSS), the well-known 10-machine 39bus New England power system is used. Its single line diagram is shown in Fig.5. A comparison with the $\mathrm{ABC}$ algorithm is carried out. All data system are taken from [22]. Optimization algorithms are implemented in MATLAB R2013a with 64-bit operating system on a PC with an Intel i7-4510U CPU @ 2.00 $\mathrm{GHz}$. For the BBO algorithm, habitat modification probability and mutation probability are chosen to be 1 and 0.01 , respectively. For fair comparison, $\mathrm{BBO}$ and $\mathrm{ABC}$ algorithms are with the same individual number and maximum number of iterations that are both fixed on 100. Different loading conditions and critical line outages have been considered in this study to test and validate the BBO-PSS controllers.

(i) Base case ;

(ii) Case 1, outage of line 21-22;

(iii) Case 2, outage of line 1-38 ;

(iv) Case 3, outage of line 21-22 with $25 \%$ increase in loads at buses 16 and 21 , and $15 \%$ increase in production of generators \#7 and \#8;

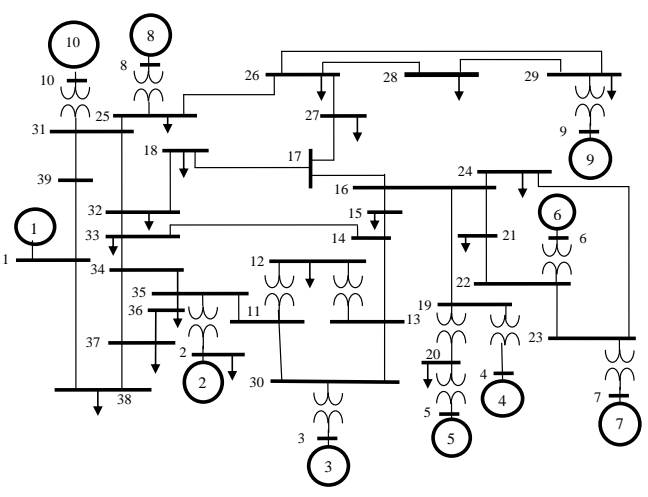

In this study, the washout time constant $T_{w}$ is fixed to $5 \mathrm{~s}$. Typical ranges of the decision variables are [0.1-50] for $K_{i}$ and [0.01-1.5] for $T_{1 i}$ to $T_{4 i}$. However, the threshold parameters of the pre-specified zone in the $s$-plane, $\sigma_{0}$ and $\xi_{0}$ are respectively -0.5 and $10 \%$.

\section{A. Optimal setting of PSS parameters}

Table I shows the system nine electromechanical modes of the studied system without PSSs for the aforementioned loading conditions. It is clear that four of them are undamped modes and the rest are lightly damped modes. Thus, it is mandatory to fix the PSS parameters in their optimum values. To reach this purpose, BBO algorithm with mutation is applied. Results obtained using the proposed algorithm are compared with those obtained using $\mathrm{ABC}$ algorithm. Fig. 6 illustrates the convergence characteristics of the two methods. The optimum PSS parameters obtained using BBO algorithm with mutation and the $\mathrm{ABC}$ are given in table II.

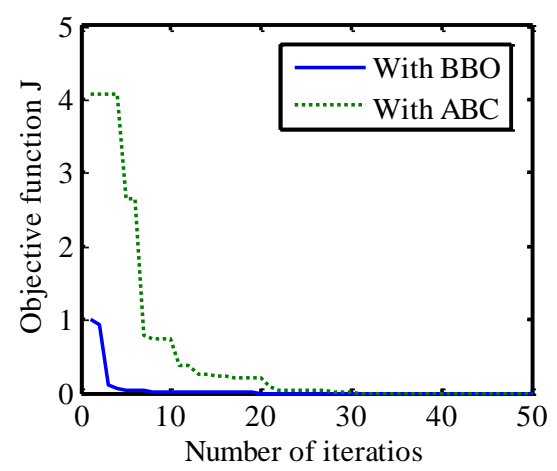

Fig. 6. Convergence characteristics

Fig. 7 shows that all electromechanical modes are shifted into the zone of the s-plane defined by $\sigma_{0}=-0.5$ and $\xi_{0}=0.1$ when BBO-PSS is applied.

Fig. 5. Single line diagram for New England system

TABLE I. EIGENVALUES AND DAMPING RATIOS WITHOUT PSSS

\begin{tabular}{cccc}
\hline \hline Base case & Case 1 & Case 2 & Case 3 \\
\hline $0.0229 \pm \mathrm{j} 3.5622, \mathbf{- 0 . 0 0 6 4}$ & $0.0891 \pm \mathrm{j} 3.3754, \mathbf{- 0 . 0 2 6 4}$ & $-0.0286 \pm \mathrm{j} 2.9442,0.0097$ & $0.1156 \pm \mathrm{j} 3.3287, \mathbf{- 0 . 0 3 4 7}$ \\
$-0.0453 \pm \mathrm{j} 5.8668,0.0077$ & $-0.0317 \pm \mathrm{j} 5.6233,0.0056$ & $-0.0252 \pm \mathrm{j} 5.8726,0.0043$ & $0.0045 \pm \mathrm{j} 5.5549, \mathbf{- 0 . 0 0 0 8}$ \\
$-0.1208 \pm \mathrm{j} 6.1132,0.0198$ & $-0.0301 \pm \mathrm{j} 5.9269,0.0051$ & $-0.1266 \pm \mathrm{j} 5.9755,0.0212$ & $-0.0370 \pm \mathrm{j} 5.9095,0.0063$ \\
$-0.1374 \pm \mathrm{j} 6.6839,0.0205$ & $-0.1284 \pm \mathrm{j} 6.1008,0.0210$ & $-0.1318 \pm \mathrm{j} 6.6695,0.0198$ & $-0.1253 \pm \mathrm{j} 6.0960,0.0205$ \\
$-0.0801 \pm \mathrm{j} 7.0358,0.0114$ & $-0.0793 \pm \mathrm{j} 6.9978,0.0113$ & $-0.0790 \pm \mathrm{j} 7.0365,0.0112$ & $-0.0800 \pm \mathrm{j} 7.0157,0.0114$ \\
$-0.1955 \pm \mathrm{j} 7.0881,0.0276$ & $-0.1843 \pm \mathrm{j} 7.0764,0.0260$ & $-0.1860 \pm \mathrm{j} 7.0776,0.0263$ & $-0.1815 \pm \mathrm{j} 7.0746,0.0256$ \\
$-0.2724 \pm \mathrm{j} 8.3551,0.0326$ & $-0.2552 \pm \mathrm{j} 8.2227,0.0310$ & $-0.2719 \pm \mathrm{j} 8.3471,0.0326$ & $-0.2406 \pm \mathrm{j} 8.2132,0.0293$ \\
$-0.2377 \pm \mathrm{j} 8.5058,0.0279$ & $-0.2718 \pm \mathrm{j} 8.3837,0.0324$ & $-0.2351 \pm \mathrm{j} 8.4968,0.0277$ & $-0.1828 \pm \mathrm{j} 8.4889,0.0215$ \\
$-0.2894 \pm \mathrm{j} 8.5178,0.0340$ & $-0.2244 \pm \mathrm{j} 8.4759,0.0265$ & $-0.2874 \pm \mathrm{j} 8.5082,0.0338$ & $-0.2184 \pm \mathrm{j} 8.5882,0.0254$ \\
\hline \hline
\end{tabular}

TABLE II. OPTIMAL PSS PARAMETERS

\begin{tabular}{|c|c|c|c|c|c|c|c|c|c|c|}
\hline \multirow[b]{2}{*}{ Gen } & \multicolumn{5}{|c|}{$B B O-P S S$} & \multicolumn{5}{|c|}{$A B C-P S S$} \\
\hline & $K$ & $T_{1}$ & $T_{2}$ & $T_{3}$ & $T_{4}$ & $K$ & $T_{1}$ & $T_{2}$ & $T_{3}$ & $T_{4}$ \\
\hline G2 & 7.53 & 0.92 & 0.09 & 0.65 & 0.04 & 34.91 & 0.13 & 0.02 & 0.84 & 0.09 \\
\hline G3 & 7.56 & 1.09 & 0.05 & 0.84 & 0.06 & 12.73 & 0.63 & 0.09 & 0.37 & 0.03 \\
\hline G4 & 13.93 & 0.97 & 0.03 & 0.10 & 0.02 & 2.54 & 0.49 & 0.09 & 1.01 & 0.01 \\
\hline G5 & 1.01 & 0.85 & 0.08 & 0.84 & 0.05 & 2.10 & 1.09 & 0.02 & 0.06 & 0.03 \\
\hline G6 & 49.44 & 0.35 & 0.10 & 1.15 & 0.04 & 9.49 & 1.40 & 0.04 & 0.05 & 0.06 \\
\hline G7 & 39.88 & 1.28 & 0.09 & 0.04 & 0.04 & 35.10 & 0.21 & 0.09 & 0.85 & 0.02 \\
\hline G8 & 46.79 & 0.38 & 0.07 & 0.53 & 0.04 & 17.31 & 0.37 & 0.08 & 0.28 & 0.06 \\
\hline G9 & 42.65 & 0.42 & 0.04 & 0.14 & 0.01 & 3.50 & 1.36 & 0.02 & 1.17 & 0.04 \\
\hline G10 & 19.36 & 1.33 & 0.09 & 0.60 & 0.09 & 35.63 & 0.43 & 0.02 & 0.54 & 0.01 \\
\hline
\end{tabular}




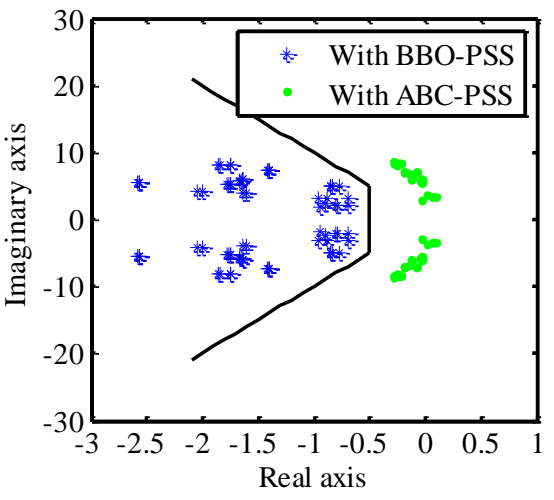

Fig. 7. Electromechanical mode

\section{B. Nonlinear analysis}

Nonlinear analysis depicted in Fig. 8 shows the effectiness of the proposed design in the improvement of the system stability over different loading conditions and its superiority compared to the ABC-based PSS design.

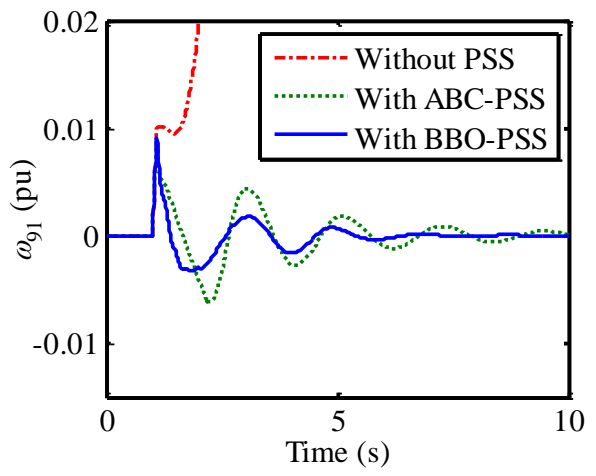

(a) For Base case

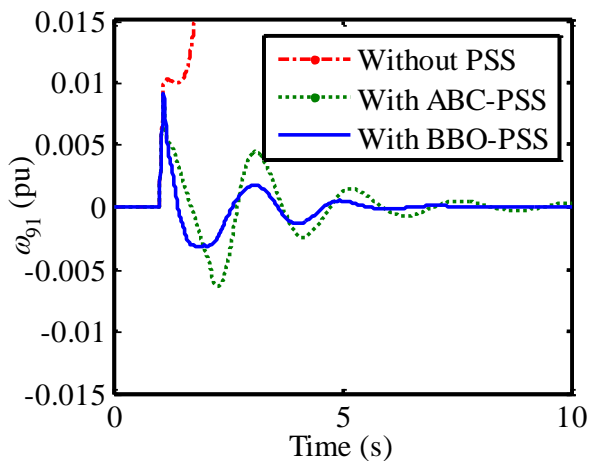

(b) For case 1

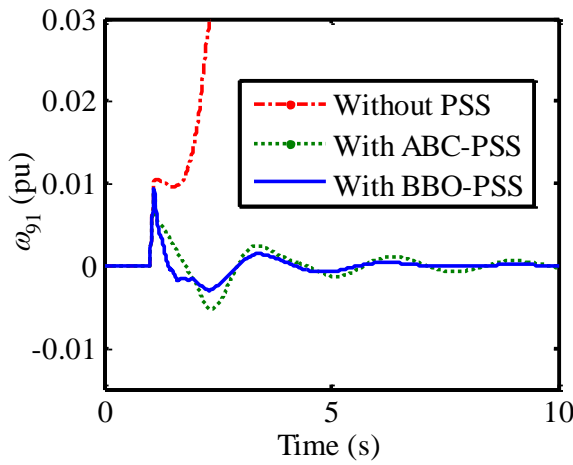

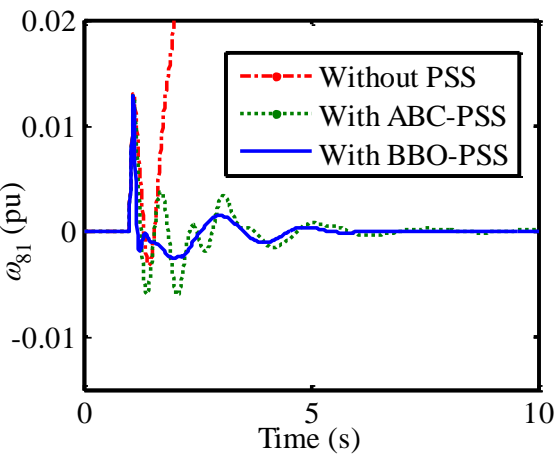

(d) For case 3

Fig. 8. Nonlinear analysis

\section{CONCLUSION}

CPSS are designed around a particular loading condition. Thus, it is not able to provide satisfactory damping over a wide range of operating conditions. To overcome this drawback, a robust multimachine PSS design was proposed in this study. The PSS design was formulated as an optimization problem in which all PSS parameters were tuned in order to shift all electromechanical modes to the left in the s-plane. The BBO algorithm with mutation operator was suggested for solving this problem. For validation, the proposed method was compared to the $\mathrm{ABC}$ algorithm, where the 10-machine 39-bus New England power system was used. Several operating conditions and configurations were used in the design problem. Simulation results showed that the speed of the search process and the stability were improved when using the proposed algorithm.

\section{REFERENCES}

[1] M.A. Abido, "Robust design of multimachine power system stabilizers using simulated annealing," IEEE Transactions on Energy Conversion, vol. 15 n. 3, 2000, pp. 297-304.

[2] P. Kundur, "Power system stability and control," New York: Tata McGraw-Hill, 1994

[3] A. Farah, T. Guesmi, H. Hadj Abdallah, "A new method for the coordinated design of power system damping controllers," Engineering Applications of Artificial Intelligence, vol. 64, 2017, pp. 325-339.

[4] D.K. Sambariya, R. Gupta, R. Prasad, "Design of optimal input-outpu scaling factors based fuzzy PSS using bat algorithm," Engineering Science and Technology, vol. 19, 2016, pp. 991-1002.

[5] M.Y. Yunus, M.R. Djalal, M. Marhatang, "Optimal design of power system stabilizer using firefly algorithm in interconnected $150 \mathrm{kV}$ sulselrabar system," Indonesia, International Review of Electrical Engineering, vol. 12 n. 3, 2017, pp. 250-259.

[6] D. K. Sambariya, R. Prasad, "Design of robust PID power system stabilizer for multimachine power system Using HS algorithm," American Journal of Electrical and Electronic Engineering, vol. 3 n. 3, 2015, pp. 75-82.

[7] D.K. Sambariya, R. Prasad, "Design of harmony search algorithm based tuned fuzzy logic power system stabilizer," International Review of Electrical Engineering, vol. 8 n. 5, 2013, pp. 1594-1607.

[8] K. Sundareswaran, S. Razia Begum, "Genetic tuning of a power system stabilizer," International Transactions on Electrical Energy Systems, vol. 14, 2004, pp. 151-160.

[9] C.Y. Chung, K.W. Wang, C.T. Tse, X.Y. Bian, A.K. David, "Probabilistic eigenvalue sensitivity analysis and PSS design in multimachine systems," IEEE Transactions on Power Systems, vol. $18 \mathrm{n}$. 4, 2003, pp. 1439-1445.

[10] J.J. Da Cruz, L.C. Zanetta, "Stabilizer design for multimachine power systems using mathematical programming," International Journal of Electrical Power and Energy Systems, vol. 19 n. 8, 1997, pp. 519-523.

(c) For case 2 
[11] K. Bollinger, A. Laha, R. Hamilton, T. Harras, "Power stabilizer design using root locus methods," IEEE Transactions on power apparatus and systems, vol.PAS-94 n. 5, September/October 1975.

[12] C.T. Tse, K.W. Wang, C.Y. Chung, K.M. Tsang, "Robust PSS design by probabilistic eigenvalue sensitivity analysis," Electric Power Systems Research, vol. 59, 2001, pp. 47-54.

[13] K. Sebaa, M. Boudour, "Optimal locations and tuning of robust power system stabilizer using genetic algorithms," Electric Power Systems Research, vol. 79, 2009, pp. 406-416.

[14] E. Babaei, S. Galvani and M. Ahmadi Jirdehi, "Design of robust power system stabilizer based on PSO," 2009 IEEE Symposium on Industrial Electronics and Applications, October 4-6, 2009, Kuala Lumpur, Malaysia.

[15] S. Ekinci, A. Demiroren, "PSO based PSS design for transient stability enhancement," IU J Electr Electron Eng, vol. 15 n. 1, 2015, pp. 18551862.

[16] M.A. Abido, Y.L. Abdel-Magid, "Eigenvalue assignments in multimachine power systems using tabu search algorithm," Computers and Electrical Engineering, vol. 28, 2002, pp. 527-545.

[17] I. Eke, M.C. Taplamacioglu, K.Y. Lee, "Robust tuning of power system stabilizer by using orthogonal learning artificial bee colony," IFACPapersOnLine, vol. 48 n. 30, 2015, pp. 149-154.

[18] S. Fang, X. Zhang, "A hybrid algorithm of particle swarm optimization and tabu search for distribution network reconfiguration," Mathematical Problems in Engineering, vol. 2016, 2016, pp. 1-7.

[19] D. Simon, "Biogeography-based optimization," IEEE Transactions on Evolutionary Computation, vol. 12, 2008, pp. 702-713.
[20] S. Habib A. Rahmati, M. Zandieh, "A new biogeography-based optimization (BBO) algorithm for the flexible job shop scheduling problem," International Journal of Advanced Manufacturing Technology, vol. 58, 2012, pp. 1115-1129.

[21] W. Gong, Z. Cai, C.X. Ling, H. Li, "A real-coded biogeography-based optimization with mutation, Applied Mathematics and Computation," vol. 216 n. 9, 2010, pp. 2749-2758.

[22] P.M. Anderson, A.A. Fouad, "Power system control and stability." 2nd Ed IEEE Press Power Engineering Series, 2003.

\section{BIOGRAPHY}

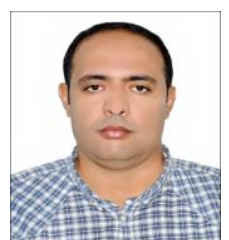

Dr Tawfik Guesmi received his $\mathrm{PhD}$ in electrical engineering from National Engineering School of Sfax, Tunisia, in 2007. His current research interests include applications of intelligent techniques for power system stability enhancement, power dispatch problems, FACTS devices and wind energy. 